\title{
Comparative Assessment of Serum versus Menstrual Blood for Diagnostic Purposes: A Pilot Study
}

\author{
Sara Naseri, Klaira Lerma, and Paul D Blumenthal* \\ Department of Obstetrics and Gynecology, School of Medicine, Stanford University, CA, USA
}

*Corresponding author: Paul Blumenthal, Pasteur Drive, Stanford, USA, Tel: 650-721-1562; E-mail: pblumen@stanford.edu

Received: 03 Oct, 2019 | Accepted: 13 Nov, 2019 | Published: 20 Nov, 2019

Citation: Naseri S, Lerma K, Blumenthal PD (2019) Comparative Assessment of Serum versus Menstrual Blood for Diagnostic Purposes: A Pilot Study. J Clin Lab Med 4(2): dx.doi.org/10.16966/2572-9578.130

Copyright: (c) 2019 Naseri S, et al. This is an open-access article distributed under the terms of the Creative Commons Attribution License, which permits unrestricted use, distribution, and reproduction in any medium, provided the original author and source are credited.

\begin{abstract}
Background: Blood testing remains an essential component of diagnosis and prevention of disease. Phlebotomists, Laboratory Infrastructure, Storage, Collection, and Transport are required for Current Methods of Blood Collection. Menstrual Blood has many similar characteristics to circulating blood but has not previously been assessed as a Potential Diagnostic Resource.
\end{abstract}

Objective: To assess if biomarkers derived from menstrual blood correlate with systemic blood.

Study design: This was a prospective, observational pilot study of healthy reproductive-aged women. We chose a panel of 9 Biomarkers, used in preventative health assessments and for following clinical conditions, and compared systemic and menstrual blood levels.

Results: Eighty-Four volunteers were screened over two months; 35 provided a menstrual and serum sample, of which 20 had a sample adequate for analysis. Overall, the correlation was observed, particularly for 7 Biomarkers, with no statistically significant differences between the mean menstrual and serum values. These include cholesterol ( $P$ for differences in means $=0.89, R 2$ for correlation=0.89), Creatinine $(P=0.32, R 2=0.94)$, HSCRP $(P=0.89, R 2=0.99)$, $L D L(P=0.21, R 2=0.84)$, Triglycerides $(P=0.45, R 2=0.89), H b a 1 c(P=0.54, R 2=0.80)$, and $\operatorname{HDL}(P=0.33, R 2=0.77)$. One biomarker, $F S H(P<0.001, R 2=0.97)$, was less directly comparable to systemic blood, but a linear relationship was recognized suggesting that the correlation could be mathematically derived, and therefore diagnostic utility is possible.

Conclusion: Based on our results, menstrual blood can reliably estimate levels of several biomarkers and may be a promising option for noninvasive collection of blood for diagnosis and health monitoring. Larger trials are needed to confirm these findings.

\section{Introduction}

Whole blood or Serum (Systemic Blood) is commonly used to diagnose or monitor many medical conditions. However, obtaining a specimen is an invasive procedure, requiring medical assistance. It can be inconvenient, costly, painful, and anxiety-provoking. Most reproductive-aged women menstruate regularly, and while menstrual blood shares many characteristics with systemic blood, it has not been subject to rigorous clinical investigations for diagnostic and therapeutic purposes.

Menstrual blood is composed of three distinct body fluids: whole blood, vaginal fluid, and the cells and fluid of the late Secretory Phase of the endometrial lining and the cervix, shed during menstruation. Molecular proteomic studies have shown considerable correlation with systemic blood; however, menstrual blood also contains additional fluids [1]. Specifically, at least 385 additional proteins can be detected in menstrual blood when compared with systemic blood [1]. In 2012 Siegel D, et al. first defined the proteomics of menstrual blood, and found that several biomarkers for a wide range of disorders were present in menstrual fluid [1]. These disorders include endometriosis, breast, Cervical, Ovarian and Endometrial Cancer. Several other studies have detected the presence of Human Papilloma Virus (HPV) in menstrual blood implying that it may be useful for non-invasive screening for cervical cancer or pre-cancer [2,3]. Furthermore, menstrual blood has been studied for screening or diagnosis of Sexually Transmitted Infections (STIS). Alary M, et al. explored the potential of using vaginal fluid collected in menstrual pads for Chlamydia detection. This proved to have sensitivity and specificity equivalent to, or even higher than, existing methods, including vaginal swabs or urine samples [4]. These data combine to provide support for the potential utility of menstrual blood-based testing as a non-invasive alternative blood source for diagnostic or therapeutic analysis.

Despite this apparent potential, menstrual blood remains a relatively uninvestigated area for diagnostics; no published literature explores the correlation of systemic versus menstrual-based blood for assessing biomarkers relative to indicators of health or disease. 
To assess this, we conducted a proof-of-concept study, comparing menstrual blood to systemic blood. We hypothesized that if menstrual blood biomarkers correlated with systemic blood, this alternative testing method could have potential as a convenient, non-invasive and cost-effective approach to blood analysis for both diagnosis and therapeutics. If found reliable, such an approach could enhance opportunities for early disease detection and regular health monitoring among women who menstruate.

\section{Materials and Methods}

This was a prospective, observational, pilot study of healthy reproductive-aged women. Interested women completed a telephone screening to assess eligibility and willingness to participate. Exclusion criteria included being younger than 18 years of age, older than 45 years, postmenopausal, not menstruating regularly, and uncomfortable with or clinically unable to use a menstrual cup for menstrual blood collection.

Those who were eligible per telephone screening were invited to an in-person meeting. During the meeting the study processes were explained in detail and instructions of how to use a menstrual cup were given to ensure participants were aware and comfortable using it for the menstrual blood collection. Demographic and other information such as age, weight, birth control usage, expected dates for menstruation, and health issues and concerns, were collected. All participants signed consent forms and were given a study kit containing a menstrual cup and two blood collection tubes. The menstrual cup (diva international inc., on, Canada) used for menstrual blood collection is a flexible menstrual cup that is worn internally, around the cervix to collect, rather than absorb, menstrual flow. It is hypo-allergenic, latex-free and safe when used as directed. The materials used in the study are commonly used and are FDA, CE or who-prequalified. The study was approved by the Stanford Institutional Review Board (IRB-35817).

Participants were instructed to contact study staff on the first day of their period, being the first day with actual flow. That day, participants were instructed to stop intake of food after midnight. On the second day of their period participants were instructed to use the menstrual cup for three hours, starting at the time they woke up in the morning, and then immediately pour the collected menstrual blood into the designated blood collection tubes. The second day was chosen due to convenience and because the second day of menstruation for most participants was found to be the day with the heaviest flow of menstrual blood. When participants presented at the study site-a venous blood draw was performed, and the menstrual blood samples were collected. The venous samples were collected on the same day as the menstrual blood samples. Neither the participants nor study staff observed any clotting in the menstrual blood samples. Both blood samples were pipetted onto Dried Blood-Spot (DBS) blood collection cards (advance DX inc., AZ, USA). DBS is considered interchangeable with venepuncture $[5,6]$ and was used as a convenient and costeffective way to transport blood samples to the laboratory for analysis. Because both samples were collected on DBS and both samples were analyzed with same methods and by the same laboratory, any effect on the samples using DBS would be applicable to both samples and therefore the comparison of the two samples is valid. A total of four cards per sample type were utilized. The eight blood collection cards per participant were shipped on the same day to a CLIA/CAP Certified Laboratory and DBS specialist, us specialty (San Diego, CA, USA), where the coded samples were analyzed. All samples were provided to the lab with no indication as to the source (i.e., menstrual or serum), so that analyses were performed in a "blinded" fashion. All participants were compensated for their travel and time spent in clinic.
Results were analyzed using paired t-tests to compare mean values of systemic blood to menstrual blood; confidence intervals were calculated to provide insight into the magnitude of the mean differences. Further, via correlation analysis of the paired samples, we analyzed whether it is possible to predict systemic blood results based on menstrual blood results. We used the method of least squares to create the trend line. Such a calculation would mean that systemic blood values are not necessarily identical to menstrual blood results, but that correlations could be mathematically predicted, indicating that certain systemic blood biomarkers can be estimated from menstrual blood. To assess the strength of the linear relationship we used Pearson correlation coefficient, which varies from -1 (perfect linear negative relationship) to +1 (perfect linear positive relationship) with values around 0 corresponding to weak relationship.

\section{Results}

Between March 2016 and March 2017, 145 volunteers were evaluated for participation, and pre-procedure meetings were arranged for 84 eligible women, who represented a convenience sample. We enrolled and collected menstrual and systemic blood from 35 participants. For 15 participants, the volume of menstrual blood collected was insufficient for analysis and those participants were excluded, leaving 20 participants in this analysis. Our participants were young and healthy, and the majority reported regular menstrual periods $[n=20$, 96.2\%] (Table 1).

In comparing paired samples of systemic blood to menstrual blood $(n=20)$, there were no statistically significant differences among values for cholesterol, FSH, HBA1C, and HSCRP. For other markers, specifically Creatinine, glucose, LDL, and triglycerides, the mean difference between the pairs was statistically significantly different; in these analyses several outliers were observed from the trend. In all cases of statistically significant differences between the paired samples, serum values were higher than menstrual values. Systemic blood demonstrated much higher levels of glucose compared to menstrual blood (Table 2).

Systemic blood values were significantly correlated with their menstrual blood counterparts $(\mathrm{p}<0.05)$, except for glucose (correlation coefficient below $0.2, p>0.05$ ). Regression analysis revealed coefficients that demonstrated a strong linear relationship for multiple analytes $(\mathrm{p}<0.05)$, table 3 , including cholesterol $(\mathrm{r}=0.942, \mathrm{p}<0.001)$, Creatinine $(\mathrm{r}=0.973, \mathrm{p}<0.001)$, FSH $(\mathrm{r}=0.982, \mathrm{p}<0.001)$, HSCRP $\mathrm{r}=0.996$, $\mathrm{p}<0.001)$, and triglycerides $(\mathrm{r}=0.944, \mathrm{p}<0.001)$. However, there was

Table 1: Participant Characteristics, $\mathrm{N}=20$.

\begin{tabular}{|c|c|}
\hline Age, mean ( \pm SD) & $28 \pm 8.7$ \\
\hline \multicolumn{2}{|c|}{ Race, $n$ (\%) } \\
\hline White & $14(70.0)$ \\
\hline Asian & $4(20.0)$ \\
\hline Other & $2(10.0)$ \\
\hline Body Mass Index (BMI), mean ( \pm SD) & $21.9 \pm 3.3$ \\
\hline Regular menstrual cycle, $n$ (\%) & $25(96.2)$ \\
\hline \multicolumn{2}{|l|}{ Hygiene product used regularly, $n(\%)$} \\
\hline Tampons only & $6(30.0)$ \\
\hline Pads only & $3(15.0)$ \\
\hline Menstrual cup & $3(15.0)$ \\
\hline Both tampons and pads & $8(40.0)$ \\
\hline
\end{tabular}


Table 2: Paired Sample Comparison of Systemic and Menstrual Blood Samples, N=20.

\begin{tabular}{|l|c|c|c|c|}
\hline \multicolumn{1}{|c|}{ Biomarker } & Systemic Blood & Menstrual Blood & Mean & \multirow{2}{*}{ 95\% Confidence Interval Of The Difference } \\
\cline { 3 - 5 } & & & Difference & 1.66 \\
\hline Cholesterol(mg/DI) & $164.86 \pm 36.84$ & $163.20 \pm 38.53$ & -0.24 & -4.38 to 7.70 \\
\hline Creatinine (mg/DI) & $1.04 \pm 0.75$ & $1.28 \pm 0.72$ & 0.13 & -0.32 to -0.16 \\
\hline FSH (Uiu/MI) & $4.99 \pm 2.33$ & $4.86 \pm 2.49$ & 80.67 & -0.09 to 0.35 \\
\hline Glucose (mg/DI) & $90.58 \pm 8.63$ & $9.91 \pm 10.30$ & 0.08 & 75.01 to 86.33 \\
\hline Hba1c (\%) & $5.35 \pm 0.36$ & $5.27 \pm 0.45$ & 3.88 & -0.02 to 0.18 \\
\hline HDL (mg/DI) & $63.10 \pm 13.27$ & $59.22 \pm 11.29$ & 0.26 & 0.92 to 6.83 \\
\hline Hscrp (mg/DI) & $4.12 \pm 6.02$ & $3.86 \pm 6.00$ & 12.12 & -0.01 to 0.51 \\
\hline LDL (mg/DI) & $95.79 \pm 30.67$ & $83.67 \pm 28.91$ & 12.49 & 6.45 to 17.78 \\
\hline Triglycerides(mg/DI) & $84.40 \pm 51.55$ & $71.91 \pm 51.12$ & 4.41 to 20.57 \\
\hline
\end{tabular}

FSH: Follicle Stimulating Hormone; HbA1c: Hemoglobin A1c; HDL: High-Density Lipoprotein; HSCRP: High Sensitivity C Reactive Protein; LDL: Low Density Lipoprotein.

Table 3: Pearson Correlation Coefficients between Menstrual and Systemic Blood Samples.

\begin{tabular}{|l|c|c|c|}
\hline & Pearson's Correlation & $\mathbf{9 5 \% ~ C l}$ & P-Value \\
\hline Cholesterol & 0.942 & $0.86-0.98$ & $<0.001$ \\
\hline Creatinine & 0.972 & $0.93-0.99$ & $<0.001$ \\
\hline FSH & 0.982 & $0.95-0.99$ & $<0.001$ \\
\hline Glucose & 0.195 & $0.27-0.59$ & 0.41 \\
\hline Hba1c & 0.892 & $0.74-0.96$ & $<0.001$ \\
\hline HDL & 0.88 & $0.71-0.95$ & $<0.001$ \\
\hline Hscrp & 0.996 & $0.99-1.00$ & $<0.001$ \\
\hline LDL & 0.919 & $0.80-0.97$ & $<0.001$ \\
\hline Triglycerides & 0.944 & $0.86-0.98$ & $<0.001$ \\
\hline
\end{tabular}

$\mathrm{Cl}$ : Confidence Interval; FSH: Follicle Stimulating Hormone; HbA1c: Hemoglobin A1c; HDL: High Density Lipoprotein; HSCRP: High Sensitivity C Reactive Protein; LDL: Low Density Lipoprotein.

poor correlation between menstrual and systemic blood for glucose $(\mathrm{r}=0.195, \mathrm{p}=0.411)$. The correlation analysis showed that although the FSH was one of the biomarkers for which we observed significant differences in systemic and menstrual blood values, using the derived equation, it was possible to predict systemic blood FSH $(r=0.982)$ knowing the result based on menstrual blood. The relationships between systemic and menstrual blood samples for some of the biomarkers are illustrated in figure 1 .

\section{Conclusion}

Our analysis indicates statistically significant correlation of values for eight biomarkers in systemic and menstrual blood samples: cholesterol, Creatinine, HSCRP, LDL, triglycerides, hbalc HDL, and FSH (Table 1). These results indicate that biomarkers in menstrual blood can approximate systemic blood levels and, based on the observations reported here, menstrual blood could be used to estimate the serum levels of these 8 biomarkers. When there was a statistically significant mean difference between systemic and menstrual blood (e.g., FSH), it was possible to generate a formula correlating the systemic blood to the menstrual blood which resulted in a high correlation coefficient. Of all the analytes tested, there was a much more marked difference between serum and menstrual blood for glucose. We hypothesize this is because the resident flora in the vagina used the glucose for their own metabolic needs, thus lowering the value relative to serum, where there are no similar resident bacteria.

While these data are promising, the data presented here is limited by a small sample size, and the stability and method of collection of menstrual blood was not optimized, leading to several failed samples. In addition, when the difference in paired means was statistically significant, the menstrual values were always lower than the serum values, indicating possible degradation of the analytes in the menstrual cup. Also, for those analytes showing statistically significant differences between the means, the difference appeared to be due to some pairs showing huge differences compared to the majority of pairs showing minor or no differences. Larger studies will likely provide better results and reference ranges for these and, possibly, other biomarkers, with outliers having less effect on the overall analysis.

Another limitation was the use of menstrual cups since 1) some women declined participation in the study because they were uncomfortable with this method of collection, and 2) it is possible that blood sitting in the menstrual cup could become degraded if left too long before analysis. A different device, such as a modified menstrual pad, could be a more convenient and comfortable method of collection and could potentially improve both participation rates and the quality of the specimen for analysis. Future studies using both larger sample sizes and utilizing an optimized system for collecting menstrual blood are needed to better establish these reference ranges as well as any adjustment formulas for relevant biomarkers. Such formulas will allow approximation of systemic blood results based on menstrual blood results with an improved degree of accuracy.

Further study is needed to establish many of the reference ranges and correlation information. We plan to expand the number of women tested to establish reference ranges and adjustment formulas for each biomarker and optimize the collection of menstrual blood, using a specially designed menstrual pad that will yield a dried blood spot for analysis instead of pooled menstrual blood in a cup.

Despite our limitations, from a preliminary, "proof of concept", perspective, these results provide data to support the potential of using menstrual blood-based testing as an alternative to systemic blood analysis. Such an alternative testing approach has the potential to change practice and potentially improve safety, convenience, and cost effectiveness of blood analysis for both diagnosis and therapeutic monitoring among menstruating women. The approach could also enhance opportunities for early detection and regular health monitoring worldwide especially in rural areas and developing countries where the population has limited access to medical professionals.

\section{Acknowledgments}

We would like to thank Professor Maecker Holden, PhD, Stanford University, Cancer Biology, and Yael Rosenberg-Hasson, 


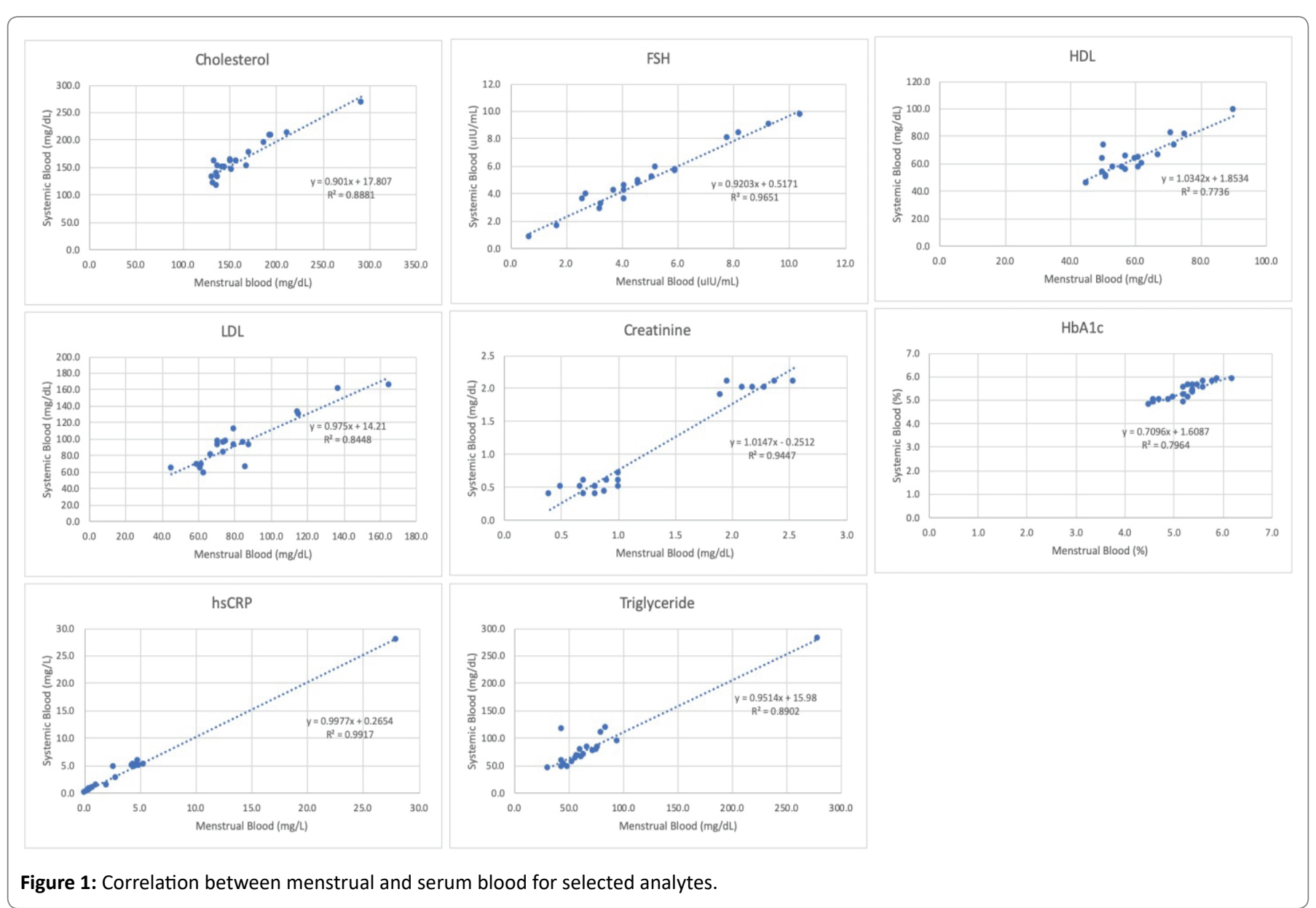

Technical Director, Institute for Immunity, Transplantation, and Infection, for their support in the initial validation phase. We also acknowledge Joel Zinda at US Specialty Labs for help with sample analysis, and Megan Fitzpatrick, MD, for careful reading of the manuscript.

\section{References}

1. Yang H, Zhou B, Prinz M, Siegel D (2012) Proteomic Analysis of Menstrual Blood. Mol Cell Proteomics 11: 1024-1035.

2. Wong SC, Au TC, Chan SC, Chan CM, Lam MY, et al. (2010) Human Papillomavirus DNA Detection in Menstrual Blood from Patients with Cervical Intraepithelial Neoplasia and Condyloma Acuminatum. J Clin Microbiol 48: 709-713.
3. Tong TR, Chan OW, Chow TC, Yu V, Leung KM, et al. (2003) Detection of Human Papillomavirus in Sanitary Napkins: A New Paradigm in Cervical Cancer Screening. Diagn Cytopathol 28: 140-141.

4. Alary M, Poulin C, Bouchard C, Fortier M, Murray G, et al. (2001) Evaluation of a Modified Sanitary Napkin as a Sample Self- Collection Device for the Detection of Genital Chlamydial Infection in Women. J Clin Microbiol 39: 2508-2512.

5. Burke EE, Beqaj S, Douglas NC, Luo R (2019) Concordance of Fingerstick and Venipuncture Sampling for Fertility Hormones. Obstet Gynecol 133: 343-348.

6. Jones TG, Warber KD, Roberts BD (2010) Analysis of Hemoglobin A1c from Dried Blood Spot Samples with the Tina-QuantR II Immunoturbidimetric Method. J Diabetes Sci Technol 4: 244-249. 